\title{
Consensuses, guidelines and position papers: the role of the Brazilian Society of Dermatology in the contribution to the best practice of Dermatology
}

DOI: http:/ /dx.doi.org/10.1590/abd1806-4841.2019940205

The Brazilian Society of Dermatology has increasingly understood and assumed its role as mediator of the discussion of the state-of-the-art and evidence-based knowledge in the practice of dermatology. The understanding of the need to make efforts for a joint action between society of specialty, academia, government and private initiative seems vital to us to effectively achieve uniform medical care for the entire Brazilian population. The construction of consensuses, guidelines and position articles based on what we have and want for the future becomes essential in the construction of a solid dermatological medical assistance.

There is still a great confusion, even in the international literature, on the construction of consensuses, guidelines and position papers although there are already quite precise published definitions. Let us see: "medical consensus" is a public statement on a particular aspect of medical knowledge that is generally agreed upon as an evidence-based, state-of-the-art knowledge by a representative group of experts in that area. ${ }^{1,2}$ A "guideline" is a statement which assists in determining a course of action. ${ }^{1}$ It aims to streamline particular processes according to sound practice. Guidelines may be issued by any organization to make the actions of its employees or divisions more predictable, and presumably of higher quality. By definition, following a guideline is never mandatory or enforced. ${ }^{1,2}$ A "position paper" is a document that presents an opinion about an issue, typically that of the author or another specified entity. Position papers are published in academia, in politics, in law and in other domains. Position papers range from the simple format of a letter to the editor through to the most complex in the form of a multi-author academic position paper. ${ }^{1}$

In this ABD supplement, the editors dare to jointly publish four consensual documents, and a guideline on diseases formerly orphaned by effective treatments. All these articles, elaborated, discussed and written by experts in the respective areas, were orchestrated and governed by the Brazilian Society of Dermatology. This effort, we hope, is only an initial seed, for a joint construction, between society, academia, government and private initiative, of an undisputed quality of medical care.

And, we understand that a centenarial medical society, solid and based on the best precepts of ethical, moral and scientific values, can and has a duty to exercise this mediation. $\square$

\section{REFERENCES}

1. Council of Europe. Developing a methodology for drawing up guidelines on best medical practice. Recommendation Rec (2001) 13 and explanatory memorandum. Strasbourg: Council of Europe Publishing; 2002.

2. De Boec, K., Castellani C., Elborn J.S., On behalf of the ECFS Board. Medical consensus, guidelines, and position papers: A policy for the ECFS. J Cyst Fibros. 2014;13:495-8 